TITLE:

\title{
Nonradiative exciton decay dynamics in hole-doped single- walled carbon nanotubes
}

\section{$\operatorname{AUTHOR}(\mathrm{S}):$}

Matsuda, Kazunari; Miyauchi, Yuhei; Sakashita, Takero; Kanemitsu, Yoshihiko

\section{CITATION:}

Matsuda, Kazunari ... [et al]. Nonradiative exciton decay dynamics in hole-doped single-walled carbon nanotubes. Physical Review B 2010, 81(3): 033409.

\section{ISSUE DATE:}

2010-01

URL:

http://hdl.handle.net/2433/93007

RIGHT:

(C) 2010 The American Physical Society 


\title{
Nonradiative exciton decay dynamics in hole-doped single-walled carbon nanotubes
}

\author{
Kazunari Matsuda, ${ }^{1, *}$ Yuhei Miyauchi, ${ }^{1,3}$ Takero Sakashita, ${ }^{1}$ and Yoshihiko Kanemitsu ${ }^{1,2}$ \\ ${ }^{1}$ Institute for Chemical Research, Kyoto University, Uji, Kyoto 611-0011, Japan \\ ${ }^{2}$ Photonics and Electronics Science and Engineering Center, Kyoto University, Kyoto 615-8510, Japan \\ ${ }^{3}$ Center for Integrated Science and Engineering, Columbia University, New York, New York 10027, USA \\ (Received 13 October 2009; revised manuscript received 27 November 2009; published 27 January 2010)
}

\begin{abstract}
We studied exciton dynamics in hole-doped single-walled carbon nanotubes (SWNTs). The intensities of photoluminescence (PL) peaks decreased and the PL lifetimes became shorter with an addition of hole dopant tetrafluorotetracyano- $p$-quinodimethane $\left(\mathrm{F}_{4} \mathrm{TCNQ}\right)$. We found that the nonradiative decay of excitons induced by the $\mathrm{F}_{4}$ TCNQ was of the order of a few tens of picoseconds for densities of $\sim 0.1 \mathrm{hole} / \mathrm{nm}$ at room temperature. Our results suggest that the nonradiative exciton relaxation in the hole-doped SWNTs can be explained by phonon-assisted indirect ionization processes.
\end{abstract}

DOI: 10.1103/PhysRevB.81.033409

PACS number(s): 78.67.Ch, 71.35.-y, 78.47.J-

The electronic and optical properties of single-walled carbon nanotubes (SWNTs) have been the subject of intense investigation for their fundamental physics interest. Since the optically excited electrons and holes are strongly confined in one-dimensional material, the electron-hole pairs form stable excitons due to the strong Coulomb interactions, and the exciton dynamics determine the optical properties in the SWNTs, even at room temperature. ${ }^{1-4}$ Radiative exciton lifetimes on the order of several nanoseconds have been measured in SWNTs at room temperature ${ }^{5}$ and these are consistent with the theoretically calculated results. ${ }^{6,7}$ However, the observed photoluminescence (PL) lifetimes are much shorter than the radiative exciton lifetimes. ${ }^{8-12}$ It follows that nonradiative decay plays important part in the exciton physics of SWNTs.

The single-layer structure of SWNTs provides a route for doping such as adsorption of molecules with high electron affinity. It is well known that SWNTs are unintentionally $p$-type under ambient conditions due to the adsorbed oxygen molecules and the performance of electronic devices are strongly affected by the doped holes. ${ }^{13,14}$ The optical properties are also sensitive to the surrounding conditions and doped carriers. ${ }^{15-18}$ Indeed, drastic changes in absorption and PL have been observed with doping using solutions of SWNTs with varying $p \mathrm{H}^{16,17}$ The understanding of the exciton dynamics, which are strongly related to these spectral changes, are quite important in the doped SWNTs, however, the detailed mechanisms of the exciton dynamics are not fully understood.

In this Brief Report, we report on the nonradiative exciton dynamics of hole-doped SWNTs from steady-state and time-resolved optical spectroscopy. Both the absorption and PL peaks of the lowest exciton state decreased in intensity with the addition of the $p$-type dopant tetrafluorotetracyano- $p$-quinodimethane $\left(\mathrm{F}_{4} \mathrm{TCNQ}\right)$. The PL decay was measured using femtosecond excitation correlation (FEC) spectroscopy, and became shorter with increasing $\mathrm{F}_{4}$ TCNQ concentration. Our experimental results indicate that the holes introduced by the $\mathrm{F}_{4} \mathrm{TCNQ}$ reduced the nonradiative exciton lifetime in SWNTs. The detailed mechanism of exciton decay via exciton-hole interactions will be discussed.

SWNTs were synthesized using the alcohol catalytic chemical vapor deposition method at $850{ }^{\circ} \mathrm{C} .{ }^{19}$ The SWNTs were isolated by dispersion in a solution of toluene with 0.07 wt\% poly[9,9-dioctylfluorenyl-2,7-diyl] (PFO), $60 \mathrm{~min}$ of moderate bath sonication, 15 min of vigorous sonication with a tip-type sonicator, and centrifugation at an acceleration of $13000 \mathrm{~g}$ for $5 \mathrm{~min}$, according to the procedure developed by Nish et al. ${ }^{20} \mathrm{~F}_{4}$ TCNQ in a solution of toluene was then added to PFO-dispersed SWNTs. ${ }^{21}$

We measured PL lifetimes using the FEC method., 10,22 The SWNTs were excited with optical pulses from a Ti:sapphire laser of central wavelength $745 \mathrm{~nm}$, repetition rate 80 $\mathrm{MHz}$, pulse duration $\sim 150 \mathrm{fs}$, and spectral width $8 \mathrm{~nm}$. The two beams separated by a delay time were chopped at 800 and $670 \mathrm{~Hz}$, respectively, and collinearly focused onto the same spot $(\sim 10 \mu \mathrm{m})$. Only the PL signal modulated at the sum frequency $(1470 \mathrm{~Hz})$ was detected using a photomultiplier and a lock-in amplifier after dispersion of the PL signal using a monochromator. The measurements were carried out with an excitation intensity of $\sim 100 \mu \mathrm{J} / \mathrm{cm}^{2}$.

Figure 1(a) shows absorption spectra of PFO-dispersed SWNTs obtained by adding the $\mathrm{F}_{4}$ TCNQ solutions. The distinct absorption spectra before adding the $\mathrm{F}_{4}$ TCNQ show that only several chiral indices are included in the PFO-dispersed SWNTs. ${ }^{20}$ The underlying background in the absorption spectrum of PFO-dispersed SWNTs is considerably sup-
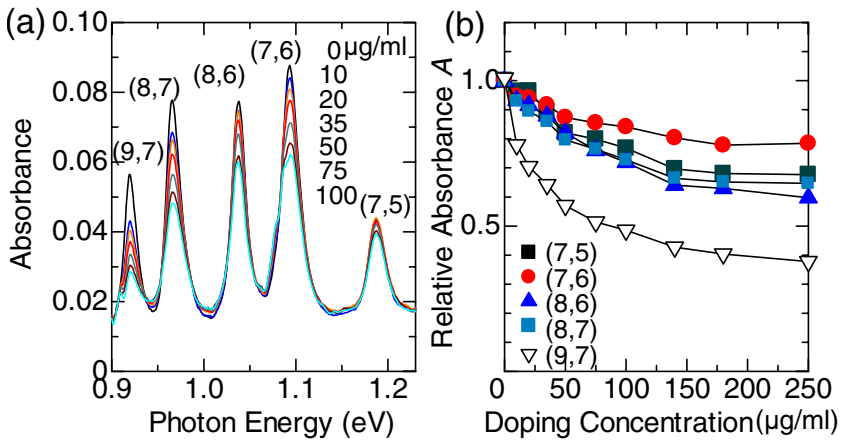

FIG. 1. (Color online) (a) Absorption spectra of PFO-dispersed SWNTs obtained with addition of $\mathrm{F}_{4}$ TCNQ solution at room temperature. (b) $\mathrm{F}_{4} \mathrm{TCNQ}$ concentration dependence of the absorption intensity normalized to the undoped intensity for each chiral index. 
(a)
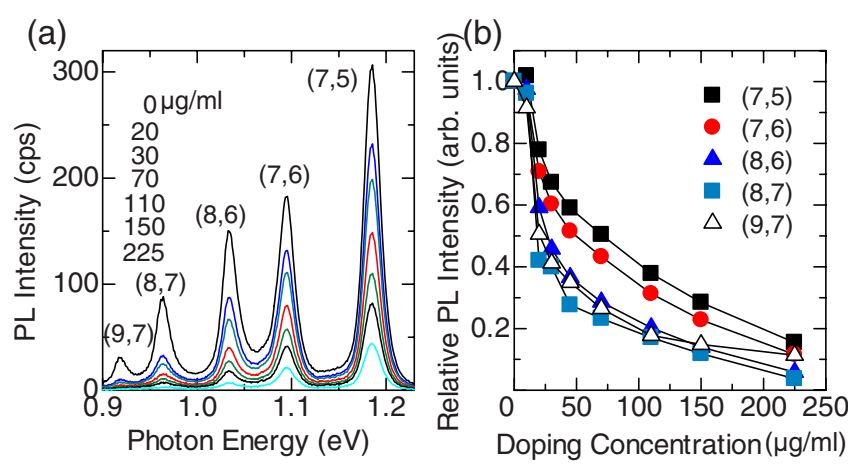

FIG. 2. (Color online) (a) PL spectra of PFO-dispersed SWNTs obtained with the addition of $\mathrm{F}_{4}$ TCNQ solution. The PL spectra were obtained with excitation at $1.96 \mathrm{eV}$. (b) PL intensities for each chiral index as functions of the $\mathrm{F}_{4} \mathrm{TCNQ}$ concentration normalized to the undoped intensity.

pressed compared to that of the standard sodium dodecyl benzene sulfonate (SDBS)-dispersed SWNTs, indicating that bundled SWNTs, and residual impurities are not included in the PFO-dispersed sample..$^{5}$ All absorption peaks decrease with the addition of $\mathrm{F}_{4}$ TCNQ molecules into the PFOdispersed SWNTs.

Figure 1(b) shows the $\mathrm{F}_{4} \mathrm{TCNQ}$ concentration dependence of the absorption intensity for each chiral index normalized to that without $\mathrm{F}_{4} \mathrm{TCNQ}$. The absorption intensity for all chiral indices decreases with increasing $\mathrm{F}_{4}$ TCNQ concentration. It decreases rapidly at first, saturating at higher concentrations. The lower energy exciton peaks from larger diameter carbon nanotubes decrease more rapidly than the higher energy peaks from the smaller diameter tubes.

Figure 2(a) shows PL spectra obtained as functions of the $\mathrm{F}_{4} \mathrm{TCNQ}$ concentration. The PL intensities for all chiral indices decrease with increasing $\mathrm{F}_{4} \mathrm{TCNQ}$ concentration. Figure 2(b) shows the PL intensities, normalized to undoped sample for each chiral index as functions of $\mathrm{F}_{4} \mathrm{TCNQ}$ concentration. The normalized PL intensities decrease more rapidly than the absorption intensities. The decrease of the normalized PL intensities depends on the chiral indices of the nanotube; the lower energy peak decreases more rapidly with increasing $\mathrm{F}_{4} \mathrm{TCNQ}$ concentration and the similar chirality dependence is also observed in the absorption spectra.

Since the electron affinity of the $\mathrm{F}_{4} \mathrm{TCNQ}$ molecules is very high, the charge transfer from the SWNTs to the $\mathrm{F}_{4}$ TCNQ occurs and SWNTs become $p$-doped. ${ }^{23-25}$ Doping SWNTs using the $\mathrm{F}_{4}$ TCNQ has been studied from the transfer characteristics of field-effect transistor ${ }^{26,27}$ and from firstprinciple calculations. ${ }^{24} \mathrm{O}^{\prime}$ Connell et al. reported a similar chirality (diameter) sensitive absorption (PL) change with the addition of $\mathrm{F}_{4} \mathrm{TCNQ}$ into SWNTs solution ${ }^{28}$ and explained the chirality (diameter) sensitivity change by the energy difference in the reduction potential of the $\mathrm{F}_{4} \mathrm{TCNQ}$ and valence band of the SWNTs.

We studied the PL decay dynamics of hole-doped SWNTs using the FEC method. The top panels of Figs. 3(a) and 3(b) show the FEC signals for undoped $(7,5)$ and $(8,6)$ SWNTs, respectively. The FEC signal is originated from the PL nonlinearities (PL saturation as a function of excitation power) (a)

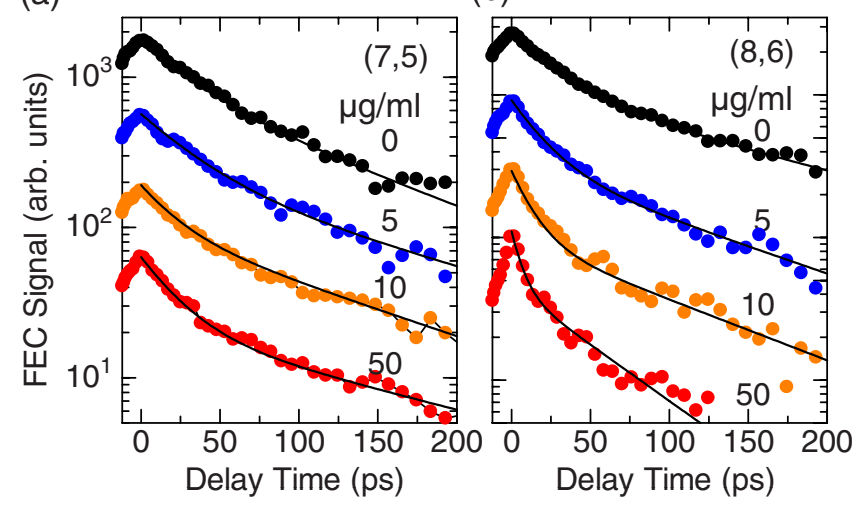

FIG. 3. (Color online) PL decay dynamics measured using the FEC method for different nanotubes, (a) $(7,5)$ and (b) $(8,6)$. The sign of the FEC decay curves is negative, and the upward direction is negative for the FEC signals. The baseline of each decay curve is shifted for clarity.

by the nonradiative exciton-exciton annihilation (Auger recombination). ${ }^{29,30}$ The decay curves show a good fit to double-exponential functions (solid lines) after subtracting the background signals. Double-exponential PL decay has recently been observed by single nanotube spectroscopy. ${ }^{12}$ The exciton population obeying double-exponential decay gives the FEC signal $I_{\mathrm{FEC}}(\tau)$ as $^{5}$

$$
I_{\mathrm{FEC}}(\tau) \propto-\left[C \tau_{\mathrm{A}} \exp \left(-\tau / \tau_{\mathrm{A}}\right)+(1-C) \tau_{\mathrm{B}} \exp \left(-\tau / \tau_{\mathrm{B}}\right)\right],
$$

where $C$ is the fractional amplitude of the fast decay component. For $(8,6)$ SWNTs, we fitted the experimental results of FEC signals and obtained $C \cong 0.90, \tau_{\mathrm{A}} \cong 30 \pm 1 \mathrm{ps}$, and $\tau_{\mathrm{B}}$ $\cong 160 \pm 6$ ps. Here we defined the effective PL lifetime as $\tau_{\mathrm{EFF}}=C \tau_{\mathrm{A}}+(1-C) \tau_{\mathrm{B}}$ and obtained $\tau_{\mathrm{EFF}}$ was $44 \pm 10 \mathrm{ps}$. The derived effective PL decay rate almost corresponds to the bright exciton decay rate $\left(\Gamma_{\mathrm{b}}\right)$.

The FEC decay curves for both $(7,5)$ and $(8,6)$ SWNTs change with the $\mathrm{F}_{4} \mathrm{TCNQ}$ concentration, as shown in Figs. $3(\mathrm{a})$ and 3(b). The changes in the decay curves are greater in the larger diameter $(8,6)$ tube and less in the smaller $(7,5)$ tube. Figure 4 shows the $\mathrm{F}_{4} \mathrm{TCNQ}$ concentration dependence of the normalized effective PL lifetimes for $(7,5),(7,6)$, and $(8,6)$ SWNTs. The effective PL lifetimes decrease with the $\mathrm{F}_{4}$ TCNQ concentration. Since the effective PL lifetimes are much shorter than the radiative exciton lifetimes in SWNTs ${ }^{5}$ and the change of radiative lifetime being proportional to inverse of the oscillator strength is small as discussed below, the effective PL lifetimes are primarily determined by nonradiative exciton decay processes. The decrease of the effective PL lifetime indicates that additional nonradiative decay paths are introduced by the doped holes. The PL intensity changes are also plotted as functions of the $\mathrm{F}_{4} \mathrm{TCNQ}$ concentration in Fig. 4(a). The change of the PL intensity is almost consistent with that of the effective PL lifetimes, which also supports that the additional nonradiative decay paths are introduced by the doped holes. The effective PL decay rate at a finite $\mathrm{F}_{4} \mathrm{TCNQ}$ concentration can be approximately described 
(a)

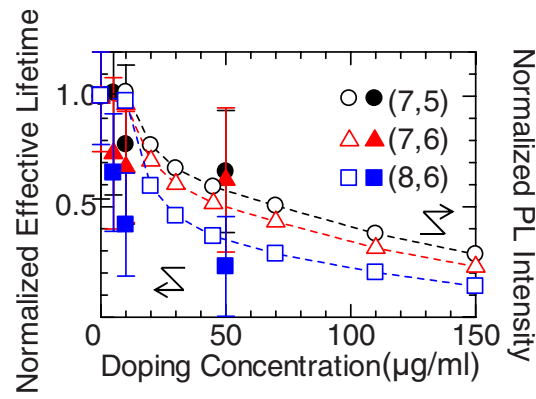

(b)

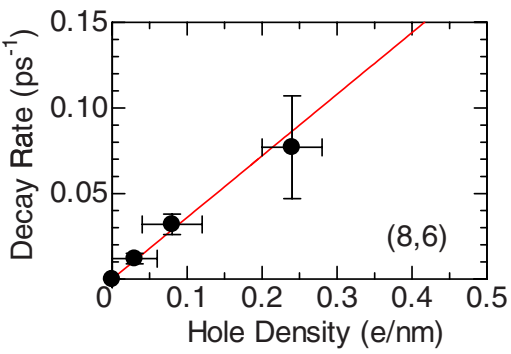

FIG. 4. (Color online) (a) Effective PL lifetimes as functions of $\mathrm{F}_{4} \mathrm{TCNQ}$ concentration. The integrated PL intensities are also shown. (b) Experimentally derived nonradiative exciton decay rates in $(8,6)$ SWNTs as functions of the hole density. The solid line is the calculated nonradiative exciton decay rates.

as $\Gamma_{\mathrm{b}}+\Gamma_{\text {hole }}$, where $\Gamma_{\mathrm{b}}$ and $\Gamma_{\text {hole }}$ are the bright exciton decay rate at undoped SWNTs, and additional nonradiative decay rate by the hole, respectively. Thus, the $\Gamma_{\text {hole }}$ can be derived from the experimentally obtained $\tau_{\mathrm{EFF}}$.

Assuming that the SWNTs are uniformly doped, we tried to roughly estimate the density of holes due to the $\mathrm{F}_{4}$ TCNQ doping from the normalized absorption change in Fig. 1(b). The exciton absorption decrease (absorption bleaching) can be explained by a state-filling effect due to holes. The exciton transition will become perfectly bleached when the Fermi energy falls below the valence band edge by an amount equal to the exciton binding energy. ${ }^{16}$ Considering the one-dimensional density of states, the density of holes in SWNTs, $N$, can be described using the corresponding hole states up to an energy $\Delta E$ from the band-edge as follows: ${ }^{16}$

$$
N=\frac{D}{\pi \hbar} \sqrt{2 m_{h} \Delta E}
$$

where $D(=4)$ is the degeneracy factor of $E_{11}$ transitions and $m_{h}$ is the effective mass for holes. We estimated that density of holes $N_{0} \sim 1.2 \mathrm{hole} / \mathrm{nm}$ for $(8,6)$ carbon nanotubes is required to perfectly bleach the exciton peak from the absorption spectrum using $m_{h}\left(=0.11 m_{0}\right.$, where $m_{0}$ is a free electron mass $)^{31}$ and exciton binding energy $\Delta E($ $=350 \mathrm{meV}) .^{32}$ The doping density at each $\mathrm{F}_{4}$ TCNQ concentration can be estimated from the absorption change using the relationship $1-A=N / N_{0}$, where $A$ is the normalized absorption intensity in Fig. 1(b).

We investigated the hole density dependence of nonradiative exciton decay rate $\Gamma_{\text {hole }}$. Figure $4($ b) shows that the derived $\Gamma_{\text {hole }}$ linearly increases as a function of the hole density. The derived $\Gamma_{\text {hole }}$ is estimated as $\sim(25 \mathrm{ps})^{-1}$ at 0.1 hole/nm for $(8,6)$ SWNTs. Recent theoretical studies have predicted the nonradiative exciton decay processes in the doped SWNTs, Auger processes involving a free hole and an exciton, ${ }^{33}$ or phonon-assisted indirect exciton ionization (PAIEI) processes. ${ }^{34}$ In the Auger process, the exciton and a free hole interact and both of the energy and momentum of the exciton are transferred to the hole. The calculated exciton-hole Auger decay rate is proportional to the Boltzmann factor, $\exp \left(-E_{\mathrm{g}} / 3 k_{\mathrm{B}} T\right)$, where $E_{\mathrm{g}}$ is the band gap, $k_{\mathrm{B}}$ is the Boltzmann constant and $T$ is temperature arising from a kinetic-energy barrier of exciton-hole recombination. ${ }^{33}$ Since excitons are created with no net momentum and all of their kinetic-energy comes from thermal fluctuations, the excitonhole Auger decay process should freeze out at low temperatures. For $(8,6)$ SWNTs with $E_{\mathrm{g}} \sim 1 \mathrm{eV}$, the calculated exciton-hole Auger decay rate should be on the order of $\sim(2 \mathrm{~ns})^{-1}$ at 0.1 hole/nm and should change by a factor of about four from 295 to $340 \mathrm{~K}$. This calculated value of the exciton-hole Auger decay rate is much different from the experimentally derived $\Gamma_{\text {hole }} \sim(25 \mathrm{ps})^{-1}$ and we confirmed that the PL lifetime does not change from 295 to $340 \mathrm{~K}$ (not shown here). Thus, the exciton-hole Auger nonradiative process is ruled out.

We considered the PAIEI process for nonradiative exciton decay. In this process, the exciton can decay by emission of a phonon, creating an intraband electron-hole pair that satisfies energy and momentum conservation. ${ }^{34}$ Since both the Coulomb and exciton-phonon interactions are enhanced in the SWNTs, the PAIEI process may occur efficiently. The nonradiative decay rate in the PAIEI process at a hole density $\rho$ is described as ${ }^{34}$

$$
\Gamma_{\text {hole }}=\frac{2 \pi}{\hbar} \frac{a S_{1} \hbar^{2} \omega^{2}}{2 \pi C d} \frac{k_{h} r_{0}}{\left(1+k_{h}^{2} r_{0}^{2}\right)^{2.6}} \frac{\rho}{E_{h}},
$$

where $S_{1}$ is an effective interband Huang-Rhys factor, $r_{0}$ is the excitonic radius as a fitting parameter, $C$ is the wave function normalization constant, $d$ is a tube diameter, $\hbar \omega$ is the $G$-mode phonon energy, $\Delta$ is the half band gap, $E_{h}$ $(=3 \Delta-\hbar \omega)$ is the hole energy, and $k_{h}=\sqrt{E_{h}-\Delta^{2}} /\left(\hbar V_{F}\right), V_{F}$ is the Fermi velocity of graphene. $a$ is determined from Ref. 34. The calculated results well reproduce the linear dependence of the nonradiative decay rate as a function of hole density and the decay rates with using the value of $r_{0}=$ $\sim 1.3 \mathrm{~nm}$ for $(8,6)$ SWNTs. The value of $r_{0}$ is physically reasonable in comparison with that from the theoretically calculated value. ${ }^{35}$ Moreover, the calculated nonradiative decay rates do not strongly depend on the temperature in the PAIEI process, which is consistent with our experimental results described above. Thus, we conclude that the exciton decay dynamics in the hole-doped SWNTs can be explained by the phonon-assisted indirect exciton ionization process.

In summary, we have reported the PL spectra and decay dynamics in the hole-doped SWNTs. The intensities of the PL peaks decreased and the PL lifetimes measured by the FEC method became shorter with increasing $\mathrm{F}_{4}$ TCNQ concentration. The densities of holes introduced by the $\mathrm{F}_{4}$ TCNQ were estimated from change in the absorption spectra. 
We found that the nonradiative decay time of excitons was about a few tens of picoseconds at a density of $\sim 0.1 \mathrm{hole} / \mathrm{nm}$ and that the nonradiative relaxation of excitons can be explained by the phonon-assisted indirect ionization process mediated via the exciton-hole interactions in SWNTs.
This study was supported by a Grant-in-Aid for Scientific Research from JSPS (Grant No. 20340075) and from MEXT of Japan (Grants No. 20048004 and No. 20104006). One of the authors (Y.M.) was financially supported by JSPS (Grant No. 20-3712). *matsuda@scl.kyoto-u.ac.jp

${ }^{1}$ F. Wang, G. Dukovic, L. E. Brus, and T. F. Heinz, Science 308, 838 (2005).

${ }^{2}$ T. Ando, J. Phys. Soc. Jpn. 66, 1066 (1997).

${ }^{3}$ H. Zhao and S. Mazumdar, Phys. Rev. Lett. 93, 157402 (2004).

${ }^{4}$ V. Perebeinos, J. Tersoff, and Ph. Avouris, Phys. Rev. Lett. 92, 257402 (2004).

${ }^{5}$ Y. Miyauchi, H. Hirori, K. Matsuda, and Y. Kanemitsu, Phys. Rev. B 80, 081410(R) (2009).

${ }^{6}$ C. D. Spataru, S. Ismail-Beigi, R. B. Capaz, and S. G. Louie, Phys. Rev. Lett. 95, 247402 (2005).

${ }^{7}$ V. Perebeinos, J. Tersoff, and Ph. Avouris, Nano Lett. 5, 2495 (2005).

${ }^{8}$ F. Wang, G. Dukovic, L. E. Brus, and T. F. Heinz, Phys. Rev. Lett. 92, 177401 (2004).

${ }^{9}$ A. Hagen, M. Steiner, M. B. Raschke, C. Lienau, T. Hertel, H. Qian, A. J. Meixner, and A. Hartschuh, Phys. Rev. Lett. 95, 197401 (2005).

${ }^{10}$ H. Hirori, K. Matsuda, Y. Miyauchi, S. Maruyama, and Y. Kanemitsu, Phys. Rev. Lett. 97, 257401 (2006).

${ }^{11}$ S. Berger, C. Voisin, G. Cassabois, C. Delalande, P. Roussignol, and X. Marie, Nano Lett. 7, 398 (2007).

${ }^{12} \mathrm{~S}$. Berciaud, L. Cognet, and B. Lounis, Phys. Rev. Lett. 101, 077402 (2008).

${ }^{13}$ P. G. Collins, K. Bradley, M. Ishigami, and A. Zettle, Science 287, 1801 (2000).

${ }^{14}$ S.-H. Jhi, S. G. Louie, and M. L. Cohen, Phys. Rev. Lett. 85, 1710 (2000).

${ }^{15}$ Y. Ohno, S. Iwasaki, Y. Murakami, S. Kishimoto, S. Maruyama, and T. Mizutani, Phys. Rev. B 73, 235427 (2006).

${ }^{16}$ G. Dukovic, B. E. White, Z. Zhou, F. Wang, S. Jockusch, M. L. Steigerwald, T. F. Heinz, R. A. Friesner, N. J. Turro, and L. E. Brus, J. Am. Chem. Soc. 126, 15269 (2004).

${ }^{17}$ G. N. Ostojic, S. Zaric, J. Kono, M. S. Strano, V. C. Moore, R. H. Hauge, and R. E. Smalley, Phys. Rev. Lett. 92, 117402 (2004).

${ }^{18}$ M. Steiner, M. Freitag, V. Perebeinos, A. Naumov, J. P. Small, A. A. Bol, and P. Avouris, Nano Lett. 9, 3477 (2009).
${ }^{19}$ S. Maruyama, R. Kojima, Y. Miyauchi, S. Chiashi, and M. Kohno, Chem. Phys. Lett. 360, 229 (2002).

${ }^{20}$ A. Nish, J.-Y. Hwang, J. Doig, and R. J. Nicholas, Nat. Nanotechnol. 2, 640 (2007).

${ }^{21}$ N. Izard, S. Kazaoui, K. Hata, T. Okazaki, T. Saito, S. Iijima, and N. Minami, Appl. Phys. Lett. 92, 243112 (2008).

${ }^{22}$ H. Hirori, K. Matsuda, and Y. Kanemitsu, Phys. Rev. B 78, 113409 (2008).

${ }^{23}$ J. Lu, S. Nagase, D. Yu, H. Ye, R. Han, Z. Gao, S. Zhang, and L. Peng, Phys. Rev. Lett. 93, 116804 (2004).

${ }^{24}$ R. G. A. Veiga and R. H. Miwa, Phys. Rev. B 73, 245422 (2006).

${ }^{25}$ It is likely that the PFO is charged by the $\mathrm{F}_{4}$ TCNQ molecules. However, we cannot observe significant lower energy shifts of the absorption and PL spectra due to the Stark effect by the charged PFO under these doping concentrations.

${ }^{26}$ T. Takenobu, T. Kanbara, N. Akima, T. Takahashi, M. Shiraishi, K. Tsukakoshi, H. Kataura, Y. Aoyagi, and Y. Iwasa, Adv. Mater. 17, 2430 (2005).

${ }^{27}$ Y. Nosho, Y. Ohno, S. Kishimoto, and T. Mizutani, Nanotechnology 18, 415202 (2007).

${ }^{28}$ M. J. O'Connell, E. E. Eibergen, and S. K. Doorn, Nature Mater. 4, 412 (2005).

${ }^{29}$ Y. Murakami and J. Kono, Phys. Rev. Lett. 102, 037401 (2009).

${ }^{30}$ Y.-F. Xiao, T. Q. Nhan, M. W. B. Wilson, and J. M. Fraser, Proc. SPIE 7201, 720111 (2009).

${ }^{31}$ A. Jorio, C. Fantini, M. A. Pimenta, R. B. Capaz, Ge. G. Samsonidze, G. Dresselhaus, M. S. Dresselhaus, J. Jiang, N. Kobayashi, A. Grüneis, and L. E. Brus, Phys. Rev. B 71, 075401 (2005).

${ }^{32}$ G. Dukovic, F. Wang, D. Song, M. Y. Sfeir, T. F. Heinz, and L. E. Brus, Nano Lett. 5, 2314 (2005).

${ }^{33}$ J. M. Kinder and E. J. Mele, Phys. Rev. B 78, 155429 (2008).

${ }^{34}$ V. Perebeinos and P. Avouris, Phys. Rev. Lett. 101, 057401 (2008).

${ }^{35}$ R. B. Capaz, C. D. Spataru, S. Ismail-Beigi, and S. G. Louie, Phys. Rev. B 74, 121401(R) (2006). 